\title{
Failure load of patellar tendon grafts at the femoral side: 10- versus 20-mm-bone blocks
}

\author{
Duncan E. Meuffels · Marnix J. N. Niggebrugge • \\ Jan A. N. Verhaar
}

Received: 8 June 2008/Accepted: 15 September 2008/Published online: 7 October 2008

(C) The Author(s) 2008. This article is published with open access at Springerlink.com

\begin{abstract}
The aim of the study was to investigate whether use of short bone blocks is safe in anterior cruciate ligament (ACL) reconstruction. Our hypothesis was that the smaller 10-mm-length bone blocks will fail at lower loads than 20-mm-bone blocks. Ten paired human cadaver knees were randomly assigned to the 10 - or 20 -mm group (group 1 and 2) and underwent bone-patellar tendon-bone femoral fixation with interference screw. Tensile tests were performed using a tensile testing machine (Instron). Stiffness, failure load and failure mode were recorded. Median stiffness was $72 \mathrm{~N} / \mathrm{mm}$ (16-103) for 10-mm-bone blocks and $91 \mathrm{~N} / \mathrm{mm}$ (40-130) for 20-mm-bone blocks. Median failure loads were $402 \mathrm{~N}$ (87-546) for 10-mm-long bone block and $456 \mathrm{~N}$ (163-636) for 20-mm-bone blocks. There was no statistically significant difference between groups $(P=0.35)$. All bone-patellar tendon-bone grafts were pulled out of the femoral tunnel with interference screw, due to slippage. We concluded that a $10-\mathrm{mm}$-long bone block was not significantly weaker than a 20-mm-long bone block. Failure loads of a 10-mm-bone block exceeded loading values at passive and active extension of the knee under normal conditions. Ten millimetre bone blocks offered sufficient fixation strength in ACL reconstruction.
\end{abstract}

Keywords Anterior cruciate ligament - Fixation · Interference screw · Pullout · Patellar tendon

D. E. Meuffels $(\bowtie) \cdot$ M. J. N. Niggebrugge · J. A. N. Verhaar Department of Orthopaedic Surgery, Erasmus MC,

University Medical Centre Rotterdam, P. O. Box 2040,

3000 CA Rotterdam, The Netherlands

e-mail: d.meuffels@erasmusmc.nl

\section{Introduction}

The bone-patellar-tendon-bone (BPTB) technique has evolved since its introduction by Jones in 1963 and by Clancy et al. in 1982 [7, 13, 14]. Jones started off with a full-length strip of patellar bone including quadriceps tendon. Clancy reduced this to a $25-\mathrm{mm}$-long bone block and we have been using this size ever since, without any scientific proof of it being the optimal length. For many years now, the bone-patella tendon-bone graft was considered the gold standard for anterior cruciate ligament (ACL) reconstruction, and is still widely used successfully despite the rising popularity of hamstring tendon reconstructions [11, 17]. However, ACL reconstruction with patellar tendon has been related to some complications, such as patellar tendinopathy, anterior knee pain, loss of range of motion and even patella fracture [6, 9, 15]. Because of these complications, attributed to harvest site morbidity, alternatives such as three- or four-stranded hamstring tendon became more and more popular. Due to the removal of a patellar bone block, there is increased strain on the patella surface, both medial and lateral to the defect [26]. Patients with a BPTB ACL reconstruction have more pain on kneeling or knee walking, compared to patients with hamstring tendon (HT) reconstruction (53 vs. $23 \%$ ) [11].

Shorter bone blocks may carry the advantage of less extensor morbidity and anterior knee pain in the postoperative period during and after rehabilitation. Theoretically, considering Steen's research, using smaller bone blocks, reduces the patellar area exposed to stress hopefully reducing anterior knee pain [26]. Furthermore, this study would show if the fixation strength would be acceptable if a surgeon decides to use a bone block which fractured peroperatively to a size of $10 \mathrm{~mm}$. 
Many studies have focused on bone block and tunnel diameter, screw size and fixation strength of different fixation devices $[1,3,10,19,25]$. These factors are very important in the first weeks of rehabilitation and different combinations have shown great variety in fixation strength. Biomechanical testing of fixation strength and failure load has been performed, mainly on fresh frozen porcine and bovine bone and some on human cadavers $[20,21,23]$.

To our knowledge, no study has been performed to establish the effects of varying length of the bone block and their failure load in interference screw fixation of ACL grafts in the human bone. The graft-femoral complex failure load will probably be determined by the bone quality rather than bone block size or be limited by the properties of the fixation device [16]. It is this complex of graft, interference screw and femur that usually leads to failure of the graft rather than the tendon rupturing itself [2].

The purpose of this study was to evaluate the difference in graft failure load between 10-and 20-mm-bone block in ACL reconstruction at the femoral fixation site. Our hypothesis is that $10-\mathrm{mm}$-bone blocks fail at lower loads than 20-mm-long bone blocks.

\section{Materials and methods}

For the present study, ten elderly paired human cadaver knee joints were used; cadavers were embalmed in formaldehyde. The knees were randomly assigned to a 10- or 20-mm-bone block reconstruction with a metal interference screw (Smith \& Nephew, MA, USA). An ACL reconstruction was performed using a 10-mm-wide patellar tendon graft with placement in a 10 -mm-diameter tunnel. The femoral ACL reconstructions are performed, creating two groups. Groups 1 and 2 had reconstruction with a bone block length of 10 and $20 \mathrm{~mm}$, respectively.

\section{Surgical technique}

A 10-mm-wide bone-patella tendon-bone graft was harvested from the cadaver using one vertical incision and an oscillating saw. Two holes were drilled in the bone block using a 1.5-mm drill; 3-0 Atraumatic Vicryl sutures (Ethicon Inc., OK, USA) were passed through to pull the graft in place at the time of screw fixation. After harvesting the graft, all soft tissue, patella and tibia were resected. The femoral tunnel was drilled over a Kirschner wire with a cannulated 10-mm drill, using a standard eleven o' clock placement for the right knee and a one o' clock position for the left knee, with a posterior wall thickness of $1 \mathrm{~mm}$. Bone blocks were made to fit using a 10-mm-diameter metal sizing tube and rongeur. The bone block was placed within the tunnel with the cortical side of the graft facing posteriorly. The $7 \times 25-\mathrm{mm}$ metal interference screw (Smith \& Nephew, MA, USA) was used for graft fixation in the femoral tunnel. The position of the bone block and interference screw in the bone tunnel was flush to the femoral intercondylar fossa.

\section{Biomechanical testing}

Failure load measurements were performed on the cadaver femurs after ACL reconstruction. Tensile testing was carried out using an Instron distraction machine (Testometric 250-2.5AX, Instron Corp., MA, USA). The specimen was cut at approximately $25 \mathrm{~cm}$ above the knee joint. The femur was positioned in the testing system so that the pulling force was parallel to the long axis of the graft. To achieve such a position, the femoral condyles were independently adjustable in the horizontal and vertical plane (Fig. 1). The distal end of the graft was fixed in a clamp with small dents assuring firm grip on the tendon. The ACL was placed under an increasing tensile load with a velocity of $1 \mathrm{~mm} / \mathrm{s}$. Measurements were performed to establish maximal stiffness, ultimate failure load and mode of failure of the patellar tendon grafts.

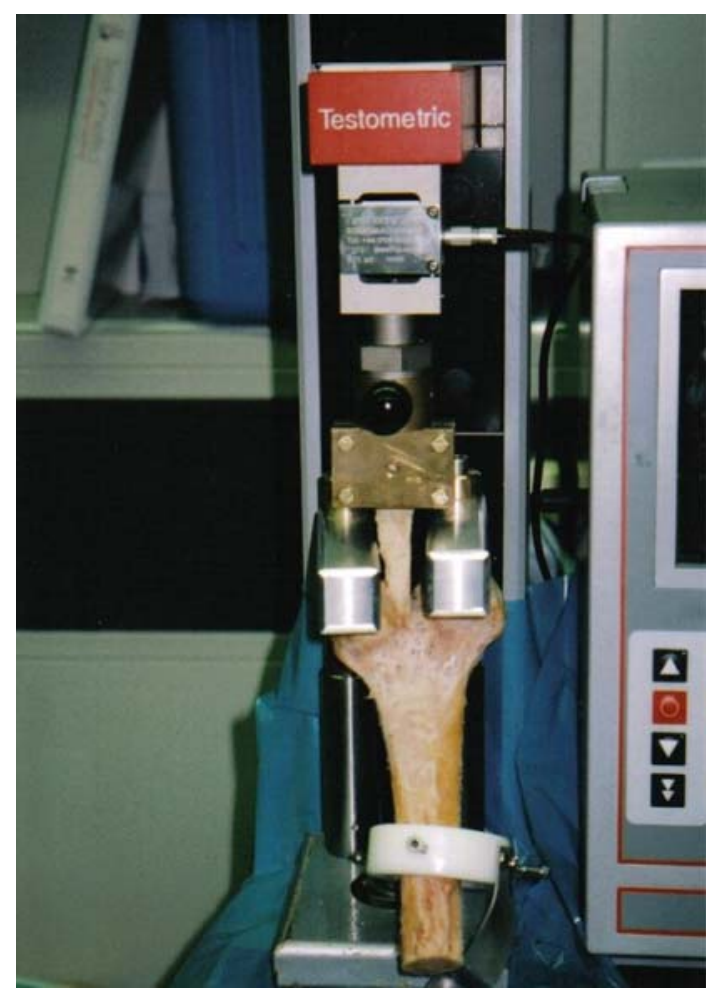

Fig. 1 Tensile testing set-up, showing adjustable femoral condyles 
Statistical analysis

The Mann-Whitney test was used to detect statistically significant differences in failure load between 10- and 20$\mathrm{mm}$-bone blocks in each cadaver. Significance was set at $P<0.05$. Data are presented using median value and range.

\section{Results}

Stiffness

Median stiffness was $72 \mathrm{~N} / \mathrm{mm}$ (16-103) for 10-mm-bone blocks and $91 \mathrm{~N} / \mathrm{mm}$ (40-130) for 20-mm-blocks. This difference was not statistically significant $(P=0.34)$ (Table 1).

Failure load

Median failure loads were $402 \mathrm{~N}$ (87-546) for 10-mm-long bone block and $456 \mathrm{~N}$ (163-636) for 20-mm-bone block without statistical significant difference $(P=0.35)$ in graft failure load between both groups (Table 2).

Failure mode

All bone-patellar tendon-bone grafts failed at the graftfemoral complex. All grafts were pulled out with screw and bone block together. All bone-tendon junctions remained intact.

\section{Discussion}

As 20-mm-bone blocks are regularly used, we tried to reduce this to $10 \mathrm{~mm}$. Our study shows no difference in failure load if we shorten the bone block by half in femoral fixation with interference screw. We have chosen for the comparison of two different lengths in bone block, since there is no known research on this topic in human cadavers.

Table 1 Stiffness of BPTB graft complex

\begin{tabular}{lll}
\hline Cadaver & $\begin{array}{l}\text { Stiffness of } \\
\text { 10-mm-bone } \\
\text { block graft complex } \\
(\mathrm{N} / \mathrm{mm})\end{array}$ & $\begin{array}{l}\text { Stiffness of 20-mm-bone } \\
\text { block graft complex } \\
\text { (N/mm) }\end{array}$ \\
\hline 1 & 55.0 & 122.3 \\
2 & 72.1 & 130.2 \\
3 & 16.3 & 39.9 \\
4 & 102.9 & 91.1 \\
5 & 92.8 & 84.6 \\
Median & 72.1 & 91.1 \\
\hline
\end{tabular}

Table 2 Failure loads of BPTB grafts

\begin{tabular}{lll}
\hline Cadaver & $\begin{array}{l}\text { Failure load } \\
10 \text {-mm-bone } \\
\text { block (N) }\end{array}$ & $\begin{array}{l}\text { Failure load } \\
20 \text {-mm-bone } \\
\text { block (N) }\end{array}$ \\
\hline 1 & 402.3 & 556.7 \\
2 & 466.1 & 635.6 \\
3 & 86.7 & 162.7 \\
4 & 545.6 & 422.7 \\
5 & 365.1 & 455.5 \\
Median & 402.3 & 455.5 \\
\hline
\end{tabular}

In both groups failure was by slippage of bone block and interference screw together. In contrast to many studies, human cadaver bone was used instead of porcine or bovine bone. We chose human bone, which is hard to come by, which resulted in a small population of higher age than the average patient undergoing an ACL reconstruction.

A clear example is our own cadaver number 3 , showing considerable weaker fixation and lower failure loads in the ACL construct. The use of elderly human cadaver bone has previously been criticised by Brown et al. [2], who showed significant differences in interference screw fixation between young and elderly human cadaver femora. Still, human cadaver femora are used for biomechanical testing of ACL reconstruction [2,5]. Our results resemble failure loads found in those studies.

Secondly, these cadavers were embalmed in formaldehyde; formaldehyde fixation of bovine bone showed almost unaffected results in loading tests [8, 29]. Nevertheless, there was a decrease in the impact strength of the bone. Forenamed factors may lead to lower values in this study than actual fixation strength in the clinical situation.

Force was applied parallel to the long axis of the graft and its tunnel. Tensile loading in this manner mimics a worst-case scenario, because it is the easiest position to pull out the graft. When pulled out at an angle, failure loads tend to be higher [24]. This would be the case when a new event happened to a patient with an ACL reconstruction.

There is a noticeable drop in the stiffness and failure load of cadaver number 3 in both the 10 - as the $20-\mathrm{mm}$ bone block. This difference is probably due to older age and more so to the low quality of the bone. Excluding this cadaver from the study would result in overall higher forces in both groups, but it also confirms that failure load depends more on bone quality in general, than on bone block length.

Rupp et al. [23] measured an ACL load of $128 \pm 15 \mathrm{~N}$ at passive and $219 \pm 25 \mathrm{~N}$ at active extension of the knee. Initial fixation strength of the graft should greatly exceed these values to make safe rehabilitation possible during the first 6 weeks. Many studies and reviews on ACL 
reconstruction have been published and up until now bonepatellar tendon-bone and hamstring tendon grafts have shown comparable results, where interference screw fixation is superior or equal to other fixation devices for initial fixation strength in BPTB ACL reconstruction [22].

The stiffness we measured is less than that of Zantop et al. and Weimann et al. $(207.2 \pm 137.5$ and $168 \pm 42$, respectively) [27, 28]. A possible explanation for these differences is the fact that both studies pulled their grafts from fresh (frozen) bovine tibia and used cross-pin fixation.

Our study shows comparable fixation strength of both bone block sizes. The failure loads are comparable to other studies with interference screw fixation $[2,4,12,18]$. This finding gives surgeons the freedom to still use a, peroperatively, fractured bone block instead of converting to contra-lateral harvesting of a second graft.

We can conclude from this study that a $10-\mathrm{mm}-$ long bone block is not significantly weaker than a 20 -mm-long bone block. Fixation strength of a 10-mm-bone block exceeds loading values at passive and active extension of the knee. Our study shows it is safe to clinically evaluate the use of smaller, shorter bone blocks, or accept intra-operative bone block fracture resulting in 10-mm-length blocks.

Acknowledgments We would like to thank Max Reijman for the statistical analysis and Jacqueline van der Linden for giving her biomechanical view. There were no sources of financial support for this study. These experiments abide the Dutch law.

Open Access This article is distributed under the terms of the Creative Commons Attribution Noncommercial License which permits any noncommercial use, distribution, and reproduction in any medium, provided the original author(s) and source are credited.

\section{References}

1. Adam F, Pape D, Steimer O, Kohn D, Rupp S (2001) Biomechanical properties of interference screw implantation in replacement of the anterior cruciate ligament with patellar and hamstring tendon transplants. An experimental study using roentgen stereometry analysis (RSA). Orthopade 30(9):649-657

2. Brown GA, Pena F, Grondvedt T, Labadie D, Engebretsen L (1996) Fixation strength of interference screw fixation in bovine, young human and elderly human cadaver knees: influence of insertion torque, tunnel-bone block gap, and interference. Knee Surg Sports Traumatol Arthrosc 3(4):238-244

3. Butler JC, Branch TP, Hutton WC (1994) Optimal graft fixation: the effect of gap size and screw size on bone plug fixation in ACL reconstruction. Arthroscopy 10(5):524-529

4. Caborn DN, Urban WP Jr, Johnson DL, Nyland J, Piekowski D (1997) Biomechanical comparison between BioScrew and titanium alloy interference screws for bone-patellar tendon-bone graft fixation in anterior cruciate ligament reconstruction. Arthroscopy 13(2):229-232

5. Camillieri G, McFarland EG, Jasper LE, Belkoff SM, Kim TK, Rauh PB, Mariani PP (2004) A biomechanical evaluation of transcondylar femoral fixation of anterior cruciate ligament grafts. Am J Sports Med 32(5):950-955
6. Christen B, Jakob RP (1992) Fractures associated with patellar ligament grafts in cruciate ligament surgery. J Bone Joint Surg Br 74(4):617-619

7. Clancy WG, Nelson DA, Reider B, Narechania RG (1982) Anterior cruciate ligament reconstruction using one-third of the patellar ligament, augmented by extra-articular tendon transfers. J Bone Joint Surg Am 64:352-359

8. Currey JD, Brear K, Zioupos P, Reilly GC (1995) Effect of formaldehyde fixation on some mechanical properties of bovine bone. Biomaterials 16:2167-2171

9. Forster MC, Forster IW (2005) Patellar tendon or four-strand hamstring? A systematic review of autografts for anterior cruciate ligament reconstruction. Knee 12(3):225-230

10. Gerich TG, Cassim A, Lattermann C, Lobenhoffer HP (1997) Pullout strength of tibial graft fixation in anterior cruciate ligament replacement with a patellar tendon graft: interference screw versus staple fixation in human knees. Knee Surg Sports Traumatol Arthrosc 5(2):84-88

11. Goldblatt JP, Fitzsimmons SE, Balk E, Richmond JC (2005) Reconstruction of the anterior cruciate ligament: meta-analysis of patellar tendon versus hamstring tendon autograft. Arthroscopy 21(7):791-803

12. Johnson LL, van Dyk GE (1996) Metal and biodegradable interference screws: comparison of failure strength. Arthroscopy 12(4):452-456

13. Jones KG (1963) Reconstruction of the anterior cruciate ligament: a technique using the central one-third of the patellar ligament. J Bone Joint Surg Am 45:925-932

14. Jones KG (1970) Reconstruction of the anterior cruciate ligament using the central one-third of the patellar ligament: a follow-up report. J Bone Joint Surg Am 52:1302-1308

15. Kartus J, Movin T, Karlsson J (2001) Donor-site morbidity and anterior knee problems after anterior cruciate ligament reconstruction using autografts. Arthroscopy 17(9):971-980

16. Krupp R, Nyland J, Smith C, Nawab A, Burden R, Caborn DN (2007) Biomechanical comparison between CentraLoc and Intrafix fixation of quadrupled semitendinosus-gracilis allografts in cadaveric tibiae with low bone mineral density. Knee 14(4):306-313

17. Laxdal G, Kartus J, Hansson L, Heidvall M, Ejerhed L, Karlsson J (2005) A prospective randomized comparison of bone-patellar tendon-bone and hamstring grafts for anterior cruciate ligament reconstruction. Arthroscopy 21(1):34-42

18. Matthews LS, Lawrence SJ, Yahiro MA, Sinclair MR (1993) Fixation strengths of patellar tendon-bone grafts. Arthroscopy 9(1):76-81

19. Milano G, Mulas PD, Ziranu F, Piras S, Manunta A, Fabbriciani C (2006) Comparison between different femoral fixation devices for ACL reconstruction with doubled hamstring tendon graft: a biomechanical analysis. Arthroscopy 22(6):660-668

20. Milano G, Mulas PD, Ziranu F, Deriu L, Fabbriciani C (2007) Comparison of femoral fixation methods for anterior cruciate ligament reconstruction with patellar tendon graft: a mechanical analysis in porcine knees. Knee Surg Sports Traumatol Arthrosc 15:733-738

21. Nadr MJ, James RV, Leung P (1993) Effect of varying angles on the pullout strength of interference screw fixation. Arthroscopy 9(5):580-583

22. Pinczewski LA, Lyman J, Salmon LJ, Russell VJ, Linklater JR, Linklater J (2007) A 10-year comparison of anterior cruciate ligament reconstructions with hamstring tendon and patellar tendon autograft: a controlled, prospective trial. Am J Sports Med 35:564-574

23. Rupp S, Hopf T, Hess T, Seil R, Kohn DM (1999) Resulting tensile forces in the human bone-patellar tendon-bone graft: direct force measurement in vitro. Arthroscopy 15:179-184 
24. Schmidt-Wiethoff R, Dargel J, Gerstner M, Schneider T, Koebke J (2006) Bone plug length and loading angle determine the primary stability of patellar tendon-bone grafts in press-fit ACL reconstruction. Knee Surg Sports Traumatol Arthrosc 14:108-111

25. Selby JB, Johnson DL, Hester P, Caborn DN (2001) Effect of screw length on bioabsorbable interference screw fixation in a tibial bone tunnel. Am J Sports Med 29(5):614-619

26. Steen H, Tseng KF, Goldstein SA, Carpenter JE (1999) Harvest of patellar tendon (bone-tendon-bone) autograft for ACL reconstruction significantly alters surface strain in the human patella. J Biomech Eng 121(2):229-233

27. Weimann A, Zantop T, Rümmler M, Hassenpflug J, Petersen W (2003) Primary stability of bone-patellar tendon-bone graft fixation with biodegradable pins. Arthroscopy 19(10):1097-1102
28. Zantop T, Rümmler M, Welbers B, Langer M, Weimann A, Petersen W (2005) Cyclic loading comparison between biodegradable interference screw fixation and biodegradable double cross-pin fixation of human bone-patellar tendon-bone grafts. Arthroscopy 21(8):934-941

29. Zech S, Goesling T, Hankemeier S, Knobloch K, Geerling J, Schultz-Brunn K, Krettek C, Richter M (2006) Differences in the mechanical properties of calcaneal artificial specimens, fresh frozen specimens and embalmed specimens in experimental testing. Foot Ankle Int 27(12):1126-1136 